\title{
The effect of antiplatelet drug on coronary endothelial and microvascular function: comparison with ticagrelor and clopidogrel
}

\author{
Woong Gil Choi ${ }^{1}$, Gi Chang Kim ${ }^{1}$, Cheol Ho Lee ${ }^{1}$, Hye Young Kim² , and Dong Woon Kim³
}

Departments of ${ }^{1}$ Internal Medicine and ${ }^{2}$ Anesthesiology and Pain Medicine, Konkuk University School of Medicine, Chungju; ${ }^{3}$ Department of Internal Medicine, College of Medicine, Chungbuk National University, Cheongju, Korea

Received: September 2, 2019 Revised : November 5, 2019 Accepted: December 29, 2019

\section{Correspondence to}

Dong Woon Kim, M.D.

Department of Internal

Medicine, College of Medicine,

Chungbuk National University,

1 Chungdae-ro, Seowon-gu,

Cheongju 28644, Korea

Tel: $+82-43-269-6386$

Fax: +82-43-269-6354

E-mail: kdwoon@chungbuk.ac.kr

https://orcid.org/0000-0002-

1988-2002
Background/Aims: Coronary endothelial and microvascular function play important roles in cardiovascular disease. We aimed to evaluate the effect of ticagrelor on coronary artery function and tested the antiplatelet effect of low dose ticagrelor in East-Asian patients.

Methods: Sixty-one consecutive patients with non-significant coronary disease were included in the study. Initially, patients were randomized in 1:1:1 ratio to receive drugs: ticagrelor $90 \mathrm{mg}$ twice a day (bid; $\mathrm{n}=22$ ), ticagrelor $45 \mathrm{mg}$ bid ( $\mathrm{n}=$ 19) or clopidogrel $75 \mathrm{mg}$ once a day (qd; $\mathrm{n}=20$ ) and then divided into two groups (ticagrelor vs clopidogrel) for evaluation of coronary artery function, and three groups for evaluation of antiplatelet function. Endothelial dysfunction was measured by coronary flow reserve (CFR), and changes in the levels of asymmetric dimethylarginine (ADMA), cluster of differentiation (CD) 40 ligand, and P-selectin. Microvascular function was evaluated as index of microvascular resistance (IMR). Platelet reactivity was assessed by VerifyNow P2Y12 assay.

Results: The levels of CFR, ADMA, and CD 40 ligand were not different between the two groups. However, P-selectin was lower in the ticagrelor group compared with clopidogrel group. IMR was significantly lower in the ticagrelor group compared with clopidogrel group (median, 15.0 [interquartile range, 12.0 to 21.0] vs. 47.5 [23.0 to 67.5$], p=0.014$ ). There was significant difference in platelet inhibition among the three groups (ticagrelor $90 \mathrm{mg}$ bid vs. ticagrelor $45 \mathrm{mg}$ bid vs. clopidogrel $75 \mathrm{mg} \mathrm{qd} ; 85.57 \pm 47.63$ vs. $120.33 \pm 51.09$ vs. $256.42 \pm 55.10, p<0.001$ )

Conclusions: It is hypothesized that ticagrelor might ameliorate the coronary microvascular function. When compared with clopidogrel, low dose ticagrelor exhibited satisfactory antiplatelet effect in the present study.

Keywords: Platelet; Microvessels; Endothelial function

\section{INTRODUCTION}

Platelets and associated antiplatelet drugs play an essential role in the pathogenesis of acute coronary syndrome and the treatment of coronary artery diseases (CADs). Over the years antiplatelet treatment has evolved to a great extent and currently several types of antiplatelet drugs are available. Ticagrelor is a potent, novel, direct-acting, and reversibly binding $\mathrm{P}_{2} \mathrm{Y}_{12}$ receptor antagonist which was approved for the treatment of patients with acute coronary syndrome [1]. Although ticagrelor and clopidogrel act on same antiplatelet target 


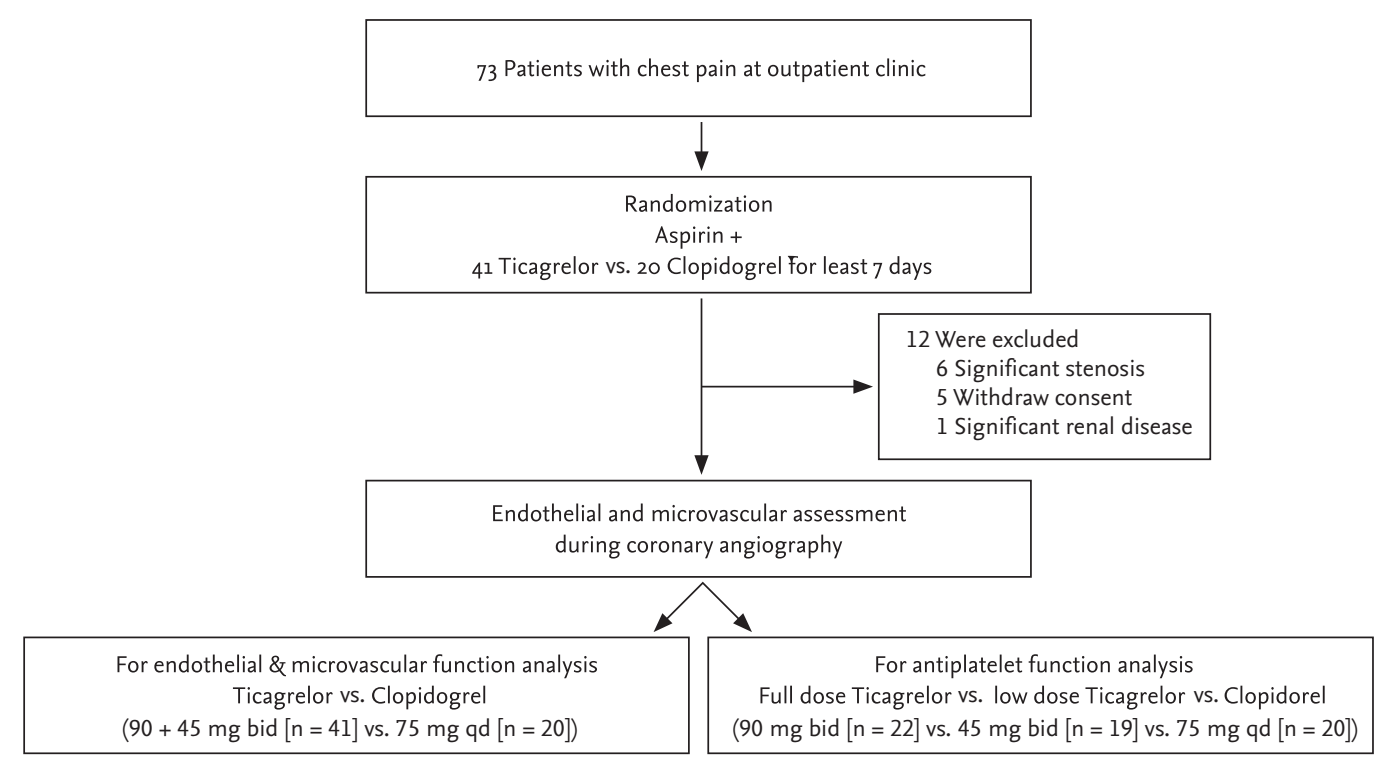

Figure 1. Actual study flow chart. bid, twice a day; qd, once a day.

receptor, ticagrelor significantly reduces the incidence of myocardial infarction, stroke, or death from vascular causes compared with standard treatment with clopidogrel in the Platelet Inhibition and Patient Outcomes trial [2]. These differences in results might not be simply explained based on the antiplatelet effect. Apparently there exists a hypothesis that ticagrelor might exhibit pleiotropic effects beyond those related to potential antiplatelet effect and that these might be relevant to its clinical efficacy.

Recent studies showed that ticagrelor treatment positively impacts endothelial function and inhibit the release of platelets' stored inflammatory mediators [3,4]. There is little data about its effect on coronary microvascular dysfunction.

In addition, Asian patients are believed to be more susceptible to bleeding, when compared with non-Asian patients [5]. Clinical pharmacology studies have reported higher levels of inhibition for platelet aggregation in Asian patients compared with Whites $[6,7]$. But, there is no data about the appropriate dose of ticagrelor, a potent antiplatelet drug, for Asian patients.

So, we aimed to evaluate the effect of ticagrelor on coronary endothelial and microvascular function in patients with CAD in comparison with effects of clopidogrel. We also tested the antiplatelet effect of low dose ticagrelor in East-Asian patients.

\section{METHODS}

\section{Study design}

This study was a single-center, open-label, and randomized controlled trial that compared the effect of ticagrelor and clopidogrel on coronary endothelial and microvascular function suggestive of worse clinical outcomes. In addition, various antiplatelet doses were evaluated for identification of appropriate effects in East-Asian patients. From May 2016 to January 2017, patients who had chest pain were enrolled from outpatient clinic at Konkuk University Chungju Hospital. Initially, patients were randomized in a $1: 1: 1$ ratio to receive drugs for at least 7 days: (1) ticagrelor $180 \mathrm{mg}$ loading and then $90 \mathrm{mg}$ twice daily $(\mathrm{n}=22),(2)$ ticagrelor $180 \mathrm{mg}$ loading and then $45 \mathrm{mg}$ twice daily ( $\mathrm{n}=19$ ), or (3) clopidogrel $300 \mathrm{mg}$ loading and then $75 \mathrm{mg}$ once a day ( $\mathrm{qd} ; \mathrm{n}=20$ ). Subsequently, the patients were divided into two groups (ticagrelor vs clopidogrel) for evaluation of endothelial and microvascular function, and into three groups (ticagrelor $90 \mathrm{mg}$ twice a day [bid] vs. ticagrelor $45 \mathrm{mg}$ bid vs. clopidogrel $75 \mathrm{mg} \mathrm{qd}$ ) for evaluation of antiplatelet function, respectively. Patients underwent laboratory testing, including assessment of endothelial function, inflammation markers, antiplatelet assay, and coronary angiography with coronary physiologic assessment (Fig. 1).

Eligibility criteria included stable angina patients old- 
er than 18 years and younger than 85 years, with non-significant (visually less than $70 \%$ stenosis and more than the value of 0.8 on fractional flow reserve) CAD. Exclusion criteria included bypass graft lesion; previous percutaneous coronary intervention (PCI) for the target vessel; heart failure; ejection fraction less than $45 \%$; myocardial infarction at the time of study enrollment; patients who required treatment with positive inotropic agents other than digoxin during the study; patients with cerebrovascular accident within 6 months before entry into the study; and significant endocrine, hepatic or renal disorders. We obtained study approval from the Institutional Review Board of Konkuk University Chungju Hospital (IRB No. KUCH 2016-02-009) and all subjects provided written informed consent.

\section{Endothelial function and inflammation marker}

Before coronary angiography, quantitative measurements of high-sensitivity C-reactive protein levels with routine laboratory tests were performed. Whole blood was centrifuged for 15 minutes at 3,000 rpm, aliquoted, and immediately frozen at $80^{\circ} \mathrm{C}$ until analysis. The markers of platelet activation and associated endothelial function (soluble cluster of differentiation [CD] 40 ligand and soluble P-selectin) were assessed using a quantitative enzyme-linked immunosorbent assay technique (Bender Med Systems GmbH, Vienna, Austria) according to the manufacturer's instructions. Additionally, the plasma concentration of asymmetric dimethylarginine (ADMA) was measured for assessment of endothelial function as previously described [8].

\section{Coronary physiologic assessment}

To assess coronary endothelial and microvascular function, we measured the index of microvascular resistance (IMR) and coronary flow reserve (CFR). Intracoronary pressure parameters in each patient were measured using previously described principles [9].

With commercially available software (Abbott Vascular Inc., Santa Clara, CA, USA), the shaft of the pressure wire can act as a proximal thermistor, sensor near the tip of the wire simultaneously measured the pressure and temperature acting as a distal thermistor. Thermo-dilution technique was used to check the transit time of room temperature when saline was injected into coronary arteries. $3 \mathrm{~mL}$ aliquots of room temperature saline

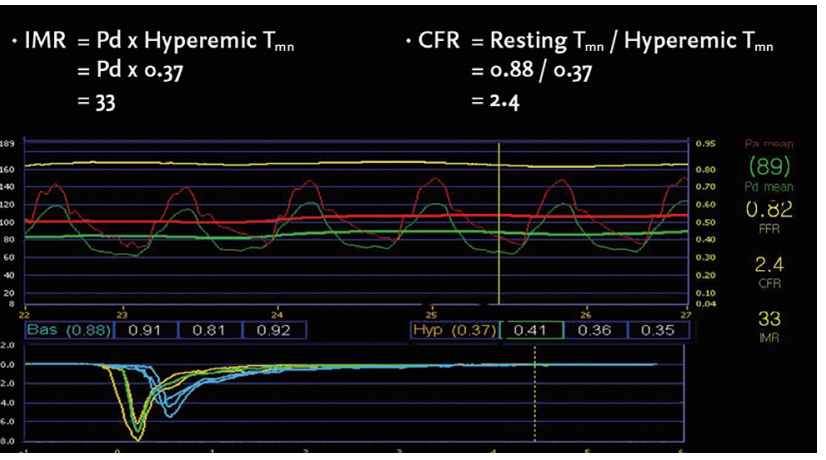

Figure 2. Calculation of index of microvascular resistance and coronary flow reserve. IMR, index of microvascular resistance; Pd, distal pressure; Tmn, mean transit time; CFR, coronary flow reserve; FFR, fractional flow reserve.

was administered to the coronary artery, and the resting mean transit time (rTmn) was measured. Steady state of maximal hyperemia was induced by intravenous infusion of adenosine $(140 \mu \mathrm{g} / \mathrm{kg} / \mathrm{min})$. Three additional $3 \mathrm{~mL}$ aliquots of room temperature saline were injected, and the hyperemic mean transit time (hTmn) was measured. The simultaneous measurements of mean aortic pressure ( $\mathrm{Pa}$, by guiding catheter) and mean distal coronary pressure (Pd, by pressure wire) were also obtained during the resting state and maximal hyperemic state (Fig. 2).

The CFR was calculated by dividing the resting hTmn by the rTmn. The IMR was defined as the simultaneously measured distal coronary pressure divided by the inverse of the hTmn (Fig. 2).

\section{Platelet function test}

Blood samples at baseline were collected via the sheath in the catheterization laboratory prior to the procedure. $\mathrm{P} 2 \mathrm{Y} 12$ reaction units (PRU) were measured using the VerifyNow assay (Accumetrics, San Diego, CA, USA). In brief, the VerifyNow assay is a whole blood, cartridge-based, optical detection system designed to measure platelet aggregation [10]. Within the cartridge of the VerifyNow $\mathrm{P}_{2} \mathrm{Y}_{12}$ assay is a channel in which inhibition of the adenosine diphosphate (ADP) $\mathrm{P}_{2} \mathrm{Y}_{12}$ receptor is measured. This channel contains ADP as a platelet agonist and prostaglandin E1 as a suppressor of intracellular-free calcium levels to reduce non-specific contribution of ADP binding to $\mathrm{P}_{2} \mathrm{Y}_{12}$ receptors, and the numerical results are expressed as PRU. 
Table 1. The baseline clinical characteristics of study groups

\begin{tabular}{lccc}
\hline Variable & Ticagrelor group $(\mathrm{n}=41)$ & Clopidogrel group $(\mathrm{n}=20)$ & $p$ value \\
\hline Male sex & $21(51)$ & $8(40)$ & 0.410 \\
Age, yr & $60.95 \pm 8.68$ & $66.85 \pm 8.52$ & 0.015 \\
Hypertension & $21(51)$ & $12(60)$ & 0.333 \\
Diabetes & $7(17)$ & $4(20)$ & 0.696 \\
Dyslipidemia & $23(56)$ & $14(70)$ & 0.214 \\
Current smoking & $4(10)$ & $1(5)$ & 0.602 \\
Stroke & $2(5)$ & $1(5)$ & 0.943 \\
Myocardial infarction & $4(10)$ & $3(15)$ & 0.487 \\
Prior medication & & & 0.393 \\
\hline Beta blocker & $11(27)$ & $7(35)$ & 0.022 \\
\hline ACE inhibitor or ARB & $12(30)$ & $11(55)$ & 0.057 \\
\hline Statin & $14(34)$ & $11(55)$ & \\
\hline
\end{tabular}

Values are presented as number (\%) or mean $\pm \mathrm{SD}$.

$\mathrm{ACE}$, angiotensin converting enzyme inhibitor; ARB, angiotensin receptor blocker.

Table 2. Laboratory finding

\begin{tabular}{lccc}
\hline Variable & Ticagrelor group $(\mathrm{n}=41)$ & Clopidogrel group $(\mathrm{n}=20)$ & $p$ value \\
\hline Hemoglobin, g/dL & $13.58 \pm 1.71$ & $13.21 \pm 1.83$ & 0.475 \\
WBC count, $10^{3} / \mu \mathrm{L}$ & $6.62 \pm 1.91$ & $6.42 \pm 1.62$ & 0.716 \\
Platelet count, $1 \mathrm{O}^{3} / \mu \mathrm{L}$ & $223.40 \pm 60.41$ & $196.11 \pm 47.58$ & 0.107 \\
Creatinine, $\mathrm{mg} / \mathrm{dL}$ & $0.81 \pm 0.17$ & $0.94 \pm 0.31$ & 0.044 \\
$\mathrm{eGFR}, \mathrm{mL} / \mathrm{min} / 1.73 \mathrm{~m}^{2}$ & $91.51 \pm 16.98$ & $81.71 \pm 22.69$ & 0.083 \\
hs-CRP, mg/dL & $0.83 \pm 1.41$ & $0.55 \pm 1.00$ & 0.525 \\
TC, $\mathrm{mg} / \mathrm{dL}$ & $176.81 \pm 44.40$ & $153.47 \pm 38.09$ & 0.077 \\
TG, $\mathrm{mg} / \mathrm{dL}$ & $148.48 \pm 95.26$ & $131.31 \pm 674.70$ & 0.525 \\
LDL-C, $\mathrm{mg} / \mathrm{dL}$ & $99.05 \pm 35.00$ & $86.00 \pm 31.18$ & 0.204 \\
HDL-C, $\mathrm{mg} / \mathrm{dL}$ & $51.08 \pm 14.93$ & $44.31 \pm 14.94$ & 0.115 \\
\hline
\end{tabular}

Values are presented as mean $\pm \mathrm{SD}$.

WBC, white blood cell; eGFR, estimated glomerular filtration rate; hs-CRP, high-sensitivity C-reactant protein; TC, total cholesterol; TG, triglyceride; LDL, low density lipoprotein cholesterol; HDL, high density lipoprotein cholesterol.

\section{Statistical methods}

All the statistical analyses were performed using SPSS version 11.0 (SPSS Inc., Chicago, IL, USA). For continuous variables, differences between the groups were evaluated by unpaired $t$ test or Mann-Whitney rank-sum test. For discrete variables, differences were expressed as counts and percentages and analyzed with chi-squared or Fisher's exact test between the groups as appropriate. A two-tailed $p$ value of 0.05 was considered to be statistically significant. Results are reported as mean \pm stan- dard deviation (SD), median (interquartile range [IQR]), or numbers (percentage).

\section{RESULTS}

\section{Baseline clinical characteristics}

The baseline clinical characteristics of patients are shown in Table 1 . The prevalence of diabetes mellitus, hypertension, hyperlipidemia and myocardial infarc- 

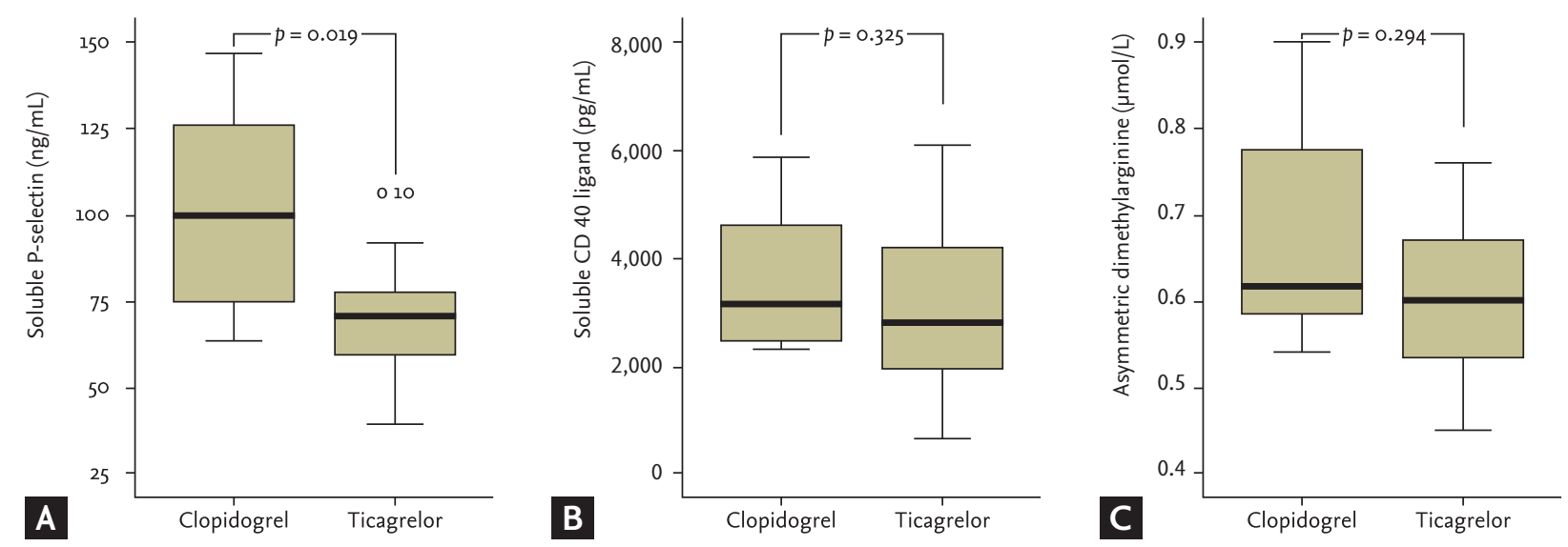

Figure 3. Comparison (A) P-selectin, (B) soluble cluster of differentiation (CD) 40 ligand, and (C) asymmetric dimethylarginine between ticagrelor group and clopidogrel group. Soluble P-selectin was significantly lower in the ticagrelor group compared with clopidogrel group, but there were no differences in the soluble CD 40 ligand and asymmetric dimethylarginine between two groups.

Table 3. Coronary angiographic and physiologic parameters

\begin{tabular}{lccc}
\hline Variable & Ticagrelor group $(\mathrm{n}=41)$ & Clopidogrel group $(\mathrm{n}=20)$ & $p$ value \\
\hline Reference diameter, mm & $3.31 \pm 0.42$ & $2.88 \pm 0.37$ & 0.028 \\
MLD diameter, mm & $1.15 \pm 0.34$ & $1.11 \pm 0.42$ & 0.839 \\
Stenosis, \% & $65.19 \pm 7.62$ & $63.16 \pm 6.47$ & 0.531 \\
Lesion length, mm & $18.59 \pm 6.24$ & $19.06 \pm 6.72$ & 0.878 \\
Target vessel & & & 0.622 \\
LAD & $30(73)$ & $15(75)$ & $1(5)$ \\
LCX & $5(12)$ & $4(20)$ & $1.85(1.13-3.60)$ \\
RCA & $6(15)$ & $0.83(0.80-0.89)$ \\
CFR & $2.0(0.7-5.5)$ & $47.5(20.75-67.50)$ \\
FFR & $0.85(0.81-0.89)$ & 0.731 \\
IMR & $15.0(12.00-21.00)$ & 0.805 \\
\hline
\end{tabular}

Values are presented as mean $\pm \mathrm{SD}$, number (\%), or median (interquartile range).

MLD, minimal luminal diameter; LAD, left anterior descending; LCX, left circumflex; RCA, right coronary artery; CFR, coronary flow reserve; FFR, fractional flow reserve; IMR, index of microvascular resistance.

tion was similar in both the groups except for age. There was no significant difference in medication except for the renin-angiotensin-aldosterone system inhibitor. There was no significant difference in total cholesterol, triglyceride, and low and high density lipoprotein cholesterol between these two groups except for the creatinine level. However, estimated glomerular filtration rate was similar in the two groups (Table 2).

\section{Endothelial and inflammation makers between tica- grelor and clopidogrel groups}

Soluble P-selectin was significantly lower in the ticagrelor group compared with clopidogrel group (70.68 [IQR, 55.20 to 80.30 ] vs. 99.65 [IQR, 70.90 to 134.78 ], $p=0.019$ ). However, there were no differences in the levels of soluble CD 40 ligand $(2,788.16$ [IQR, 1,914.31 to $4,276.39$ ] vs. 3,145.95 [IQR, 2,407.01 to 4,745.52], $p=0.325$ ) and ADMA (o.6o [IQR, 0.53 to 0.67 ] vs. 0.62 [IQR, 0.58 to 0.81$], p=$ 0.294 ) between the two groups (Fig. 3). In the subgroup 

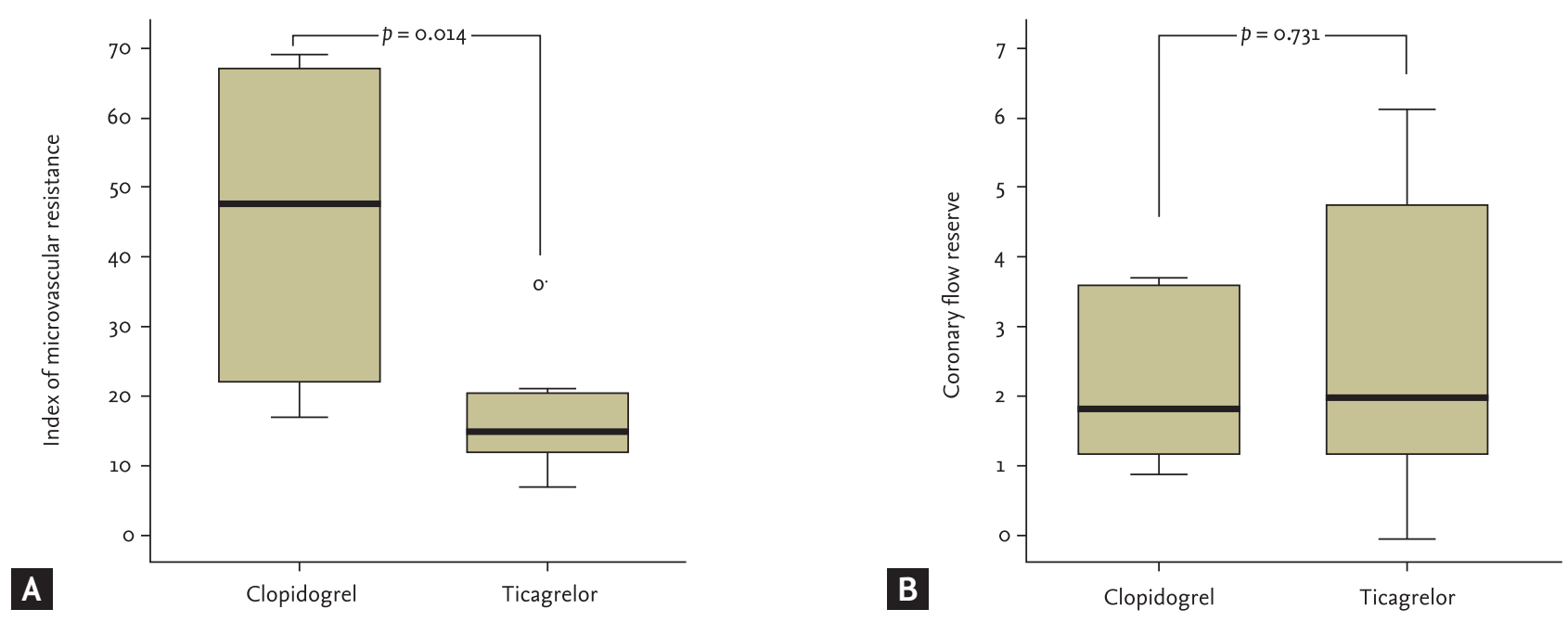

Figure 4. Comparison (A) index of microvasuclar resistance and (B) coronary flow reserve between ticagrelor and clopidogrel group. Index of microvascular resistance was significantly lower in the ticagrelor group; however, coronary flow reserve was similar between both groups.

analysis, there were no differences in the levels of CD 40 ligand, P-selectin and ADMA among the three groups (ticagrelor $90 \mathrm{mg}$ bid vs. ticagrelor $45 \mathrm{mg}$ bid vs. clopidogrel $75 \mathrm{mg} q d ; p=0.077$ for P-selectin, $p=0.574$ for $\mathrm{CD} 40$ ligand, $p=0.752$ for ADMA).

\section{Coronary angiographic characteristics and coronary physiologic assessment}

The angiographic parameters, fractional flow reserve (FFR), CFR and IMR are shown in Table 3. Angiographic characteristics were comparable in both the groups except for the reference diameter. The diameter of the patient group receiving ticagrelor was larger compared with the patients receiving clopidogrel. There was no difference in the FFR between both the groups. Fig. 4 illustrates comparison of CFR and IMR between the two groups. CFR was not significantly different in both the groups (2.0 [IQR, 0.7 to 5.5] vs.1.85 [IQR, 1.13 to 3.6o], $p=$ 0.731 ). However, IMR was significantly lower in the patient group receiving ticagrelor than clopidogrel group (15.0 [IQR, 12.00 to 21.00 ] vs. 47.5 [IQR, 20.75 to 67.50 ], $p=$ 0.014). IMR was widely distributed with higher level in the clopidogrel group compared with the ticagrelor group.

\section{Platelet function test among the three groups}

There were significant differences among the three groups with regards to platelet function (ticagrelor 90 mg bid, $85.57 \pm 47.63$ vs. ticagrelor $45 \mathrm{mg}$ bid, $120.33 \pm 51.09$ vs. clopidogrel $75 \mathrm{mg} \mathrm{qd}, 256.42 \pm 55.10, p<0.001$ ) (Fig. 5).

Although the ticagrelor $90 \mathrm{mg}$ bid group had the strongest antiplatelet function among the three groups, platelet inhibition of ticagrelor $45 \mathrm{mg}$ bid group was also significantly stronger than that of clopidogrel group suggestive of sufficient dose for East-Asian patients (Fig. 5).

In the association between platelet function and adhesion molecule, soluble P-selectin was significantly correlated with antiplatelet function $(r=0.529, p=0.014)$ (Fig. 6).

\section{DISCUSSION}

To the best of our knowledge, this is first study to simultaneously compare coronary endothelial and microvascular functions between patients with ticagrelor and those with clopidogrel. In our study, the administration of ticagrelor significantly improved coronary microvascular function whereas no effect was observed in endothelial function.

There were several reports that antiplatelet drugs could affect endothelial or vascular function. Clopidogrel or prasugrel improves endothelial nitric oxide bioavailability and diminishes biomarkers of oxidant stress and in- 


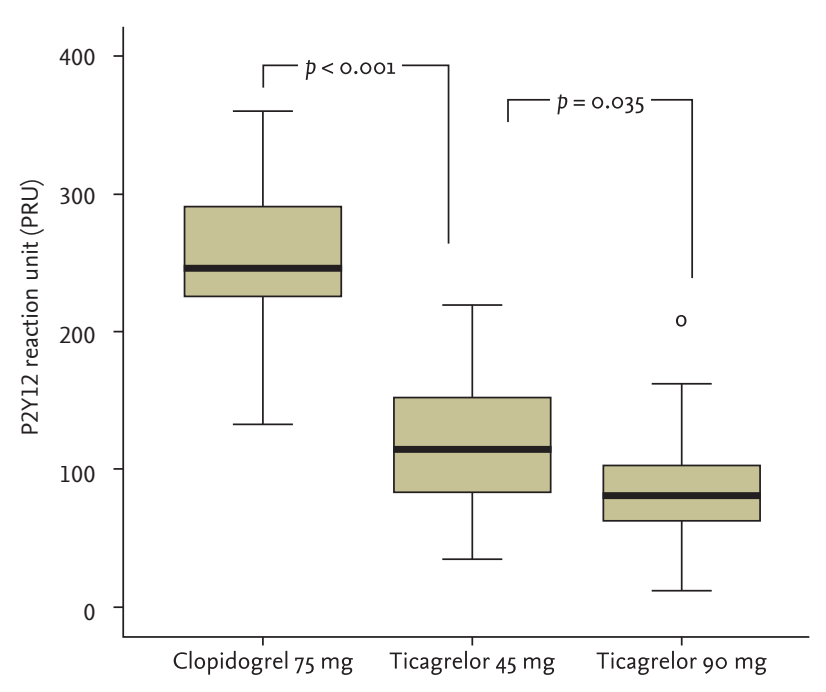

Figure 5. Comparison antiplatelet function among ticagrelor $90 \mathrm{mg}$ twice a day (bid), ticagrelor $45 \mathrm{mg}$ bid and clopidogrel group. Although ticagrelor $90 \mathrm{mg}$ bid group had the strongest antiplatelet function among the three groups, platelet inhibition of ticagrelor $45 \mathrm{mg}$ bid group was also significantly stronger than that of clopidogrel group. PRU, $\mathrm{P} 2 \mathrm{Y}_{12}$ reaction unit.

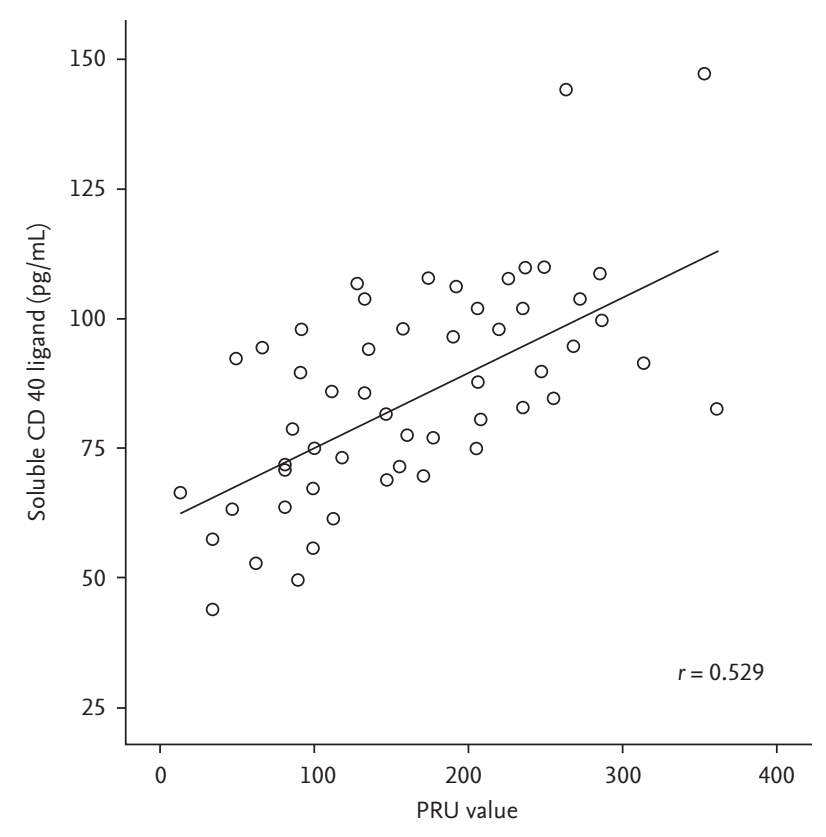

Figure 6. Association between platelet function and P-selectin. Soluble P-selectin was significantly correlated with antiplatelet function. $\mathrm{PRU}, \mathrm{P}_{2} \mathrm{Y}_{12}$ reaction unit.

flammation in patients with CAD, suggesting that beyond inhibition of platelet aggregation, adenosine phosphate receptor blockade may also have promising vasoprotective effects [11,12]. A recent study showed that ticagrelor induced significant increase in circulating endothelial progenitor cells, contributing to improved arterial endothelial function in diabetic non-ST-segment elevation acute coronary syndrome patients compared with prasugrel [13].

However, the association among endothelial marker, microvascular function and antiplatelet drugs in patients with CAD has not been studied. In the present study, we demonstrated that ticagrelor was considerably associated with coronary microvascular function based on assessment by IMR but not endothelial function by CFR or biomarker. This result is inconsistent compared with the results of previous studies [11,13-16]. These observations might be explained as follows: we included patients with relatively stable disease state and insignificant stenosis. Apparently, our absolute hs-CRP level was relatively low compared with other studies even though atherosclerosis is generally an inflammatory process. P-selectin and soluble CD 40 ligands are from different expression cells but similar clinical properties. P-selectin is a single chain glycoprotein of $140 \mathrm{kDa}$ expressed in mostly platelets and endothelial cells but soluble CD 40 ligand, which is shed from stimulated lymphocytes and is actively released after platelet stimulation $[17,18]$. Consequently soluble CD 40 ligand was considered as an independent risk marker of cardiovascular events in acute coronary syndrome $[19,20]$. In contrast, increased level of soluble P-selectin has been reported in a wide variety of acute and chronic cardiovascular conditions [21,22]. Therefore, we assumed that P-selectin was significantly lower by potent antiplatelet drug in our patient population with stable state compared to CD 40 ligand.

Consideration the relationship between CFR and IMR, our results showed discordance between CFR and IMR even though CFR reflects coronary microvascular function. The previous study also showed about 30\% discordance between CFR and IMR in high FFR patients and described the clinical relevance of IMR and CFR is unclear [23]. In addition, CFR can be highly variable according to the hemodynamic dependence of basal flow. So, it is difficult to define and maintain true resting conditions during CFR measurement. In contrast, IMR was known that it provides a more reproducible assessment of the microcirculation than CFR [9]. 
It is well known that the presence and the degree of microvascular and endothelial dysfunctions are related to the risk of subsequent cardiovascular events [24,25]. In our study, the coronary microvascular function was better in the ticagrelor group compared with clopidogrel group. The coronary microcirculation to regulate myocardial perfusion is regulated myocardial metabolic activity and endothelium-dependent vasodilation effectively and efficiently [26]. In our study, IMR and P-selectin levels were lower in the ticagrelor group. ADMA and CD 40 ligand were comparable between the two groups. Although further studies are needed for elucidating the exact mechanism [27], ticagrelor is suggested as an antiplatelet drug, as it might affect microvascular function through platelet-endothelial dependent pathway but not anti-inflammatory pathway.

We also evaluated platelet function for a low dose of ticagrelor for Asian patients, who are believed to be more susceptible to bleeding. In the results, even the low dose ticagrelor showed significantly stronger antiplatelet function compared with standard clopidogrel dose similar to other studies $[28,29]$. It is proposed that low dose of ticagrelor impart a more benefit-risk balance than ticagrelor $90 \mathrm{mg}$ bid or clopidogrel for East-Asian patients.

This study has several limitations. First, in the present study, the population studied was small, and further investigations with a larger population are required in future studies. Second, the antiplatelet medication interval was relatively short. Most of the patients underwent treatment for 7 days. It is hypothesized that ticagrelor could demonstrate better results for endothelial markers if administered longer period. However, Gao et al. [30] evaluated the endothelial function with ticagrelor administration for 7 days. In another study, microvascular function was evaluated after just $180 \mathrm{mg}$ loading dose [31]. Third, drug cross-over was not designed. Under conditions of cross-over for antiplatelet drugs, the effect of each drug on microvascular and endothelial functions could be clarified. Lastly, reference diameter was bigger in ticagrelor group compared with clopidogrel group and we measured IMR for intermediate stenotic artery. However, minimal microvascular resistance does not change with epicardial stenosis severity [32,33]. In contrast, another study showed IMR significantly decreased after PCI [34]. In our study, cases with visually significant stenosis were excluded.

The results reveal that ticagrelor might ameliorate the coronary microvascular function. This effect could explain the reason for ticagrelor being superior to clopidogrel in randomized controlled trial. In addition, when compared with clopidogrel, low dose ticagrelor exhibited sufficient antiplatelet effect. Evaluation of antiplatelet effect on coronary endothelial function is necessitated in the future studies. Low dose ticagrelor is proposed as an alternative antiplatelet treatment instead of full dose ticagrelor in East-Asian patients

\section{KEY MESSAGE}

1. Ticagrelor might ameliorate coronary microvascular function.

2. Low dose ticagrelor has sufficient antiplatelet effect in East-Asian patients.

\section{Conflict of interest}

No potential conflict of interest relevant to this article was reported.

\section{Acknowledgments}

This paper was written as part of Konkuk University's research support program for its faculty on sabbatical leave in 2016.

\section{REFERENCES}

1. Van Giezen JJ, Nilsson L, Berntsson P, et al. Ticagrelor binds to human $\mathrm{P} 2 \mathrm{Y}(12)$ independently from ADP but antagonizes ADP-induced receptor signaling and platelet aggregation. J Thromb Haemost 2009;7:1556-1565.

2. Wallentin L, Becker RC, Budaj A, et al. Ticagrelor versus clopidogrel in patients with acute coronary syndromes. $\mathrm{N}$ Engl J Med 2009;361:1045-1057.

3. Wittfeldt A, Emanuelsson H, Brandrup-Wognsen G, et al. Ticagrelor enhances adenosine-induced coronary vasodilatory responses in humans. J Am Coll Cardiol 2013;61:723-727.

4. Sumaya W, Storey RF. Ticagrelor: effects beyond the P2Y(12) receptor. Interv Cardiol Clin 2017;6:49-55.

5. Misumida N, Aoi S, Kim SM, Ziada KM, Abdel-Latif A. 
Ticagrelor versus clopidogrel in East Asian patients with acute coronary syndrome: systematic review and meta-analysis. Cardiovasc Revasc Med 2018;19:689-694.

6. Li J, Tang W, Storey RF, Husted S, Teng R. Population pharmacokinetics of ticagrelor in patients with acute coronary syndromes. Int J Clin Pharmacol Ther 2016;54:666674.

7. Lee JH, Ahn SG, Park B, et al. A pharmacodynamic study of the optimal $\mathrm{P}_{2} \mathrm{Y}_{12}$ inhibitor regimen for East Asian patients with acute coronary syndrome. Korean J Intern Med 2015;30:620-628.

8. Stühlinger MC, Abbasi F, Chu JW, et al. Relationship between insulin resistance and an endogenous nitric oxide synthase inhibitor. JAMA 2002;287:1420-1426.

9. Ng MK, Yeung AC, Fearon WF. Invasive assessment of the coronary microcirculation: superior reproducibility and less hemodynamic dependence of index of microcirculatory resistance compared with coronary flow reserve. Circulation 2006;113:2054-2061.

10. van Werkum JW, van der Stelt CA, Seesing TH, Hackeng CM, ten Berg JM. A head-to-head comparison between the VerifyNow $\mathrm{P}_{2} \mathrm{Y}_{12}$ assay and light transmittance aggregometry for monitoring the individual platelet response to clopidogrel in patients undergoing elective percutaneous coronary intervention. J Thromb Haemost 2006;4:2516-2518.

11. Heitzer T, Rudolph V, Schwedhelm E, et al. Clopidogrel improves systemic endothelial nitric oxide bioavailability in patients with coronary artery disease: evidence for antioxidant and antiinflammatory effects. Arterioscler Thromb Vasc Biol 2006;26:1648-1652.

12. Rudolph TK, Fuchs A, Klinke A, et al. Prasugrel as opposed to clopidogrel improves endothelial nitric oxide bioavailability and reduces platelet-leukocyte interaction in patients with unstable angina pectoris: a randomized controlled trial. Int J Cardiol 2017;248:7-13.

13. Jeong HS, Hong SJ, Cho SA, et al. Comparison of ticagrelor versus prasugrel for inflammation, vascular function, and circulating endothelial progenitor cells in diabetic patients with non-ST-segment elevation acute coronary syndrome requiring coronary stenting: a prospective, randomized, crossover trial. JACC Cardiovasc Interv 2017;10:1646-1658.

14. Husted S, Storey RF, Harrington RA, Emanuelsson H, Cannon CP. Changes in inflammatory biomarkers in patients treated with ticagrelor or clopidogrel. Clin Cardiol
2010;33:206-212.

15. Palmerini T, Barozzi C, Tomasi L, et al. A randomized study comparing the antiplatelet and antiinflammatory effect of clopidogrel $150 \mathrm{mg}$ /day versus $75 \mathrm{mg} /$ day in patients with ST-segment elevation acute myocardial infarction and poor responsiveness to clopidogrel: results from the DOUBLE study. Thromb Res 2010;125:309-314.

16. Braun OO, Johnell M, Varenhorst C, et al. Greater reduction of platelet activation markers and platelet-monocyte aggregates by prasugrel compared to clopidogrel in stable coronary artery disease. Thromb Haemost 2008;100:626633.

17. Blann AD, Nadar SK, Lip GY. The adhesion molecule P-selectin and cardiovascular disease. Eur Heart J 2003;24:2166-2179.

18. Graf D, Muller S, Korthauer U, van Kooten C, Weise C, Kroczek RA. A soluble form of TRAP (CD4O ligand) is rapidly released after $\mathrm{T}$ cell activation. Eur J Immunol 1995;25:1749-1754.

19. Varo N, de Lemos JA, Libby P, et al. Soluble CD4oL: risk prediction after acute coronary syndromes. Circulation 2003;108:1049-1052.

20. Dominguez-Rodriguez A, Abreu-Gonzalez P, Garcia-Gonzalez MJ, Kaski JC. Soluble CD40 ligand:interleukin-10 ratio predicts in-hospital adverse events in patients with ST-segment elevation myocardial infarction. Thromb Res 2007;121:293-299.

21. O'Connor CM, Gurbel PA, Serebruany VL. Usefulness of soluble and surface-bound P-selectin in detecting heightened platelet activity in patients with congestive heart failure. Am J Cardiol 1999;83:1345-1349.

22. Parker C 3rd, Vita JA, Freedman JE. Soluble adhesion molecules and unstable coronary artery disease. Atherosclerosis 2001;156:417-424.

23. Lee JM, Jung JH, Hwang D, et al. Coronary flow reserve and microcirculatory resistance in patients with intermediate coronary stenosis. J Am Coll Cardiol 2016;67:11581169.

24. Schachinger V, Britten MB, Zeiher AM. Prognostic impact of coronary vasodilator dysfunction on adverse longterm outcome of coronary heart disease. Circulation 2000;101:1899-1906.

25. Cohn JN, Quyyumi AA, Hollenberg NK, Jamerson KA. Surrogate markers for cardiovascular disease: functional markers. Circulation 2004;109(25 Suppl 1):IV31-IV46.

26. Herrmann J, Kaski JC, Lerman A. Coronary microvascular 
dysfunction in the clinical setting: from mystery to reality. Eur Heart J 2012;33:2771-2782b.

27. Ariotti S, Ortega-Paz L, van Leeuwen M, et al. Effects of ticagrelor, prasugrel, or clopidogrel on endothelial function and other vascular biomarkers: a randomized crossover study. JACC Cardiovasc Interv 2018;11:1576-1586.

28. Xue HJ, Shi J, Liu B, et al. Comparison of half- and standard-dose ticagrelor in Chinese patients with NSTE-ACS. Platelets 2016;27:440-445.

29. Liu GZ, Zhang S, Sun DH, et al. Half-dose ticagrelor versus high-dose clopidogrel in reducing platelet reactivity in acute coronary syndrome patients with high on-clopidogrel platelet reactivity (divide study). Eur J Clin Pharmacol 2019;75:1059-1068.

30. Gao CZ, Ma QQ, Wu J, et al. Comparison of the effects of ticagrelor and clopidogrel on inflammatory factors, vascular endothelium functions and short-term prognosis in patients with acute ST-segment elevation myocardial infarction undergoing emergency percutaneous coro- nary intervention: a pilot study. Cell Physiol Biochem 2018;48:385-396.

31. Park SD, Lee MJ, Baek YS, et al. Randomised trial to compare a protective effect of Clopidogrel Versus TIcagrelor on coronary Microvascular injury in ST-segment Elevation myocardial infarction (CV-TIME trial). EuroIntervention 2016;12:e964-e971.

32. Aarnoudse W, Fearon WF, Manoharan G, et al. Epicardial stenosis severity does not affect minimal microcirculatory resistance. Circulation 2004;110:2137-2142.

33. Fearon WF, Kobayashi Y. Invasive assessment of the coronary microvasculature: the index of microcirculatory resistance. Circ Cardiovasc Interv 2017;10:e005361.

34. Murai T, Kanaji Y, Yonetsu T, et al. Preprocedural fractional flow reserve and microvascular resistance predict increased hyperaemic coronary flow after elective percutaneous coronary intervention. Catheter Cardiovasc Interv 2017;89:233-242. 